\title{
Flexão estática e massa específica aparente da madeira de Inga marginata em diferentes condições de umidade
}

\author{
Luana Candaten $^{1 *(\mathbb{D}) \text {, Edivane Francielli Rodrigues }}{ }^{1}$ (D), Rômulo Trevisan ${ }^{1}$ (D), Élder Eloy'iD, Mágda Rosa Fontoura1(D) \\ ${ }^{1}$ Universidade Federal de Santa Maria, BR 386, Km 40, Linha Sete de Setembro, s/n, CEP 98400-000, Frederico Westphalen, RS, Brasil
}

"Autor correspondente:

luana_candaten@outlook.com

Termos para indexação:

Condicionamento humidade da madeira

Resistência mecânica

Propriedade físico-química

Index terms:

Wood conditioning

Mechanical resistance

Chemicophysical propertie

Histórico do artigo:

Recebido em 16/07/2018

Aprovado em 12/12/2018

Publicado em 29/12/2018

doi: 10.4336/2018.pfb.38e201801671

\begin{abstract}
Resumo - O presente estudo teve como objetivo determinar a flexão estática e a massa específica aparente da madeira de Inga marginata em diferentes condições de umidade. Foram abatidos três indivíduos com, aproximadamente, seis anos de idade. Para a determinação das propriedades estudadas foram seguidas as recomendações da norma ASTM D143-94. Observou-se redução superior a 1,2 vezes do módulo de elasticidade, módulo de ruptura, tensão no limite proporcional e força máxima na flexão estática em função da saturação das fibras. De maneira geral, as amostras apresentaram valores de massa específica aparente menores e maior resistência mecânica conforme a dessorção até a umidade de equilíbrio de $12 \%$, sendo a madeira, nessa condição, classificada como moderadamente pesada.
\end{abstract}

\section{Static bending and apparent specific gravity of Inga marginata wood in different humidity conditions}

\begin{abstract}
The present study aimed to determine the static bending and the apparent specific gravity of Inga marginata wood in different humidity conditions. Three individuals were cut at an approximate age of six years. For determination of the studied properties, we followed the recommendations of ASTM D143-94 norm. We observed reduction of more than 1.2 times the modulus of elasticity, modulus of rupture, tension at the proportional limit and maximum force in the static bending as a function of fiber saturation. In general, the samples presented smaller apparent specific gravity values and higher mechanical resistance according to the desorption until the equilibrium moisture of $12 \%$. The wood, in this condition, was classified as moderately heavy.
\end{abstract}

Inga marginata, popularmente conhecida por ingámirim, angá, ingá, ingá-amendoin, ingá-dedo, ingáfeijão, ingá-miúdo, ingaí e ingazeiro é classificada, segundo Moriwaki et al. (2017), como nativa da Mata Atlântica, sendo normalmente indicada para recuperação de matas ciliares. $\mathrm{O}$ estudo com madeiras naturalmente desenvolvidas no país é importante em função da escassa literatura disponível, principalmente, quando se trata das características tecnológicas das espécies.

Devido à sua natureza anisotrópica e higroscópica, a caracterização da variação mecânica desse material exige o conhecimento de seus atributos dependentes de umidade, uma vez que, em muitas aplicações, é exposta a diferentes condições climáticas (Ozyhar et al., 2012; Almeida et al., 2016).

Um dos principais indicadores da qualidade da madeira é a massa específica (Bonduelle et al., 2015; Eleotério et al., 2015), pois essa propriedade está associada à espécie, procedência da árvore, local de amostragem na tora e umidade (Trevisan et al., 2012). Além de ser uma característica de fácil determinação, é praticamente 
indispensável, uma vez que pode afetar o comportamento do material em relação à trabalhabilidade, estabilidade dimensional, durabilidade natural e resistência mecânica (Braz et al., 2015).

A massa específica está relacionada às propriedades mecânicas do material as quais podem ser avaliadas por ensaios ou testes, como por exemplo de flexão estática, permitindo comparações com as informações de espécies já estudadas e caracterizadas na literatura. Assim, devido à importância de estudos com espécies nativas e o conhecimento de suas propriedades, o objetivo desse trabalho foi determinar a flexão estática e a massa específica aparente da madeira de Inga marginata Willd. em diferentes condições de umidade.

Foram amostradas três árvores de I. marginata com, aproximadamente, seis anos de idade, situadas na Universidade Federal de Santa Maria, Campus Frederico Westphalen $\left(27^{\circ} 23^{\prime} 46,85^{\prime}\right.$ 'S e $\left.53^{\circ} 25^{\prime} 40,66^{\prime \prime} \mathrm{W}\right)$ com altitude de $462 \mathrm{~m}$. Após o abate, foi selecionada, de cada um dos indivíduos, a primeira tora com 1,40 m de comprimento. Essas foram transportadas até a serraria para a confecção de 40 corpos de prova com dimensões de $2,5 \times 2,5 \times 41 \mathrm{~cm}$, seguindo a norma D143-94 (American Society for Testing and Materials, 1995). No Laboratório de Tecnologia e Utilização de Produtos Florestais da UFSM/FW, vinte corpos de prova ficaram submersos em um tanque com água e o restante permaneceu em câmara climatizada até alcançar a umidade de equilíbrio de $12 \%$.

As informações sobre as características mecânicas da espécie submetida ao ensaio de flexão estática foram obtidas para as amostras nas condições saturada e com teor de umidade de equilíbrio a 12\%. Para tal, foi utilizada uma máquina universal de ensaios de materiais (EMIC) dotada de sistema automatizado de aquisição de dados, sendo determinadas as variáveis: força máxima $\left(\mathrm{F}_{\text {máx }}\right)$, módulo de elasticidade (MOE), módulo de ruptura (MOR) e tensão no limite proporcional $\left(f_{\mathrm{LP}}\right)$, de acordo com a ASTM D143-94 (American Society for Testing and Materials, 1995).

Após a obtenção dos dados de flexão estática e massa específica aparente, os mesmos foram avaliados por análise de variância para delineamento inteiramente casualizado através do pacote "Statgraphics Plus" e, em caso de rejeição da hipótese de igualdade de médias, utilizou-se o teste T - DMS (Diferença Mínima Significativa, $\alpha=5 \%$ ). A correlação de Pearson entre as variáveis estudadas foi determinada com o auxílio do software Statistical Analysis System (SAS Institute, 2003), com $1 \%$ de probabilidade de erro.

Todos os valores obtidos a partir dos ensaios de flexão estática tiveram redução na condição saturada quando comparada à condição seco ao ar a $12 \%$ de umidade (Tabela 1). A maior alteração foi verificada na propriedade mecânica de tensão no limite proporcional em função da elevada razão x/y, enquanto a menor variação dos dados observados ocorreu no módulo de elasticidade. A propriedade física de massa específica aparente apresentou o comportamento esperado, ou seja, houve uma diminuição nos valores conforme a dessorção até a umidade de equilíbrio de $12 \%$.

Tabela 1. Análise de variância e teste de comparação de médias de flexão estática e massa específica aparente na condição saturada e a $12 \%$ de umidade para Inga marginata.

Table 1. Analysis of variance and mean comparison test of static bending and apparent specific gravity in saturated condition and at 12\% moisture content for Inga marginata.

\begin{tabular}{|c|c|c|c|c|c|}
\hline \multirow[b]{2}{*}{ Variável } & \multicolumn{2}{|c|}{ Condição } & \multirow[b]{2}{*}{$\begin{array}{c}\text { Razão } \\
(x / y)\end{array}$} & \multirow[b]{2}{*}{ Fcal } & \multirow[b]{2}{*}{$\begin{array}{l}\text { Prob. } \\
>\text { F }\end{array}$} \\
\hline & $\begin{array}{c}\text { Seco a } 12 \%(x) \\
\left(S_{y x}\right)\end{array}$ & $\begin{array}{c}\text { Saturado }(y) \\
\left(S_{y x}\right)\end{array}$ & & & \\
\hline $\mathrm{F}_{\text {máx }}$ & $\begin{array}{l}164,7 \mathrm{a} \\
( \pm 8,2)\end{array}$ & $\begin{array}{c}134,6 \mathrm{~b} \\
( \pm 8,2)\end{array}$ & 1,22 & 6,75 & $0,0132 *$ \\
\hline MOR & $\begin{array}{c}660,5 \mathrm{a} \\
( \pm 170,3)\end{array}$ & $\begin{array}{c}481,3 \mathrm{~b} \\
( \pm 125,5)\end{array}$ & 1,37 & 14,35 & $0,0005^{* *}$ \\
\hline $\mathrm{MOE}$ & $\begin{array}{c}67259,4 \mathrm{a} \\
( \pm 15551,8)\end{array}$ & $\begin{array}{c}55799,3 \mathrm{~b} \\
( \pm 17871,9)\end{array}$ & 1,20 & 4,58 & 0,0391 * \\
\hline TLP & $\begin{array}{l}396,8 \mathrm{a} \\
( \pm 88,7)\end{array}$ & $\begin{array}{l}266,3 \mathrm{~b} \\
( \pm 74,1)\end{array}$ & 1,50 & 25,51 & $0,0001^{* *}$ \\
\hline $\mathrm{MEa}$ & $\begin{array}{c}0,576 \mathrm{a} \\
( \pm 0,055)\end{array}$ & $\begin{array}{c}1,161 \mathrm{~b} \\
( \pm 0,055)\end{array}$ & 0,50 & 1146,8 & $0,0001^{* *}$ \\
\hline
\end{tabular}

$\mathrm{F}_{\text {máx }} .=$ carga máxima aplicada $(\mathrm{Kgf}) ; \mathrm{MOE}=$ módulo de elasticidade

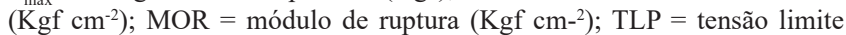
proporcional $\left(\mathrm{Kgf} \mathrm{cm}^{-2}\right) ; \mathrm{MEa}=$ massa específica aparente na condição de umidade analisada $\left(\mathrm{g} \mathrm{cm}^{-3}\right) ; \mathrm{S}_{y x}=$ erro padrão da estimativa; Prob $>\mathrm{F}=$ nível de probabilidade de erro; $* \mathrm{e}^{* *}=$ significativo ao nível de $5 \%$ e $1 \%$ de probabilidade de erro, respectivamente. Médias seguidas de letras diferentes na linha diferem estatisticamente pelo teste T - DMS (Diferença mínima significativa, $\alpha=5 \%$ ).

A madeira de ingá, na condição saturada, apresentou menores valores de elasticidade e resistência, bem como da força máxima aplicada e da tensão no limite proporcional (Tabela 1), estando em conformidade com os resultados observados por Haselein et al. (2002) e Cezaro et al. (2016), os quais relataram que abaixo do ponto de saturação das fibras (PSF) há um decréscimo dessas características com o aumento da umidade no material. Da mesma forma, Kollmann \& Côté (1968) observaram melhoria na propriedade mecânica à medida 
que houve redução na quantidade de água na madeira, resultado do fortalecimento, rigidez dos elementos estruturais e da sua compactação, em virtude da contração que está associada a dessorção.

Essa relação nas propriedades mecânicas da madeira é recorrente, pois, com a diminuição da quantidade de água na estrutura abaixo do PSF, ou seja, aproximadamente $30 \%$ de umidade, a variação das características tende a ser linear, ao contrário de quando está acima desse ponto, onde a resistência permanece praticamente constante e inalterada (Almeida et al., 2016).

Diferentes espécies florestais nessas condições de umidade foram estudadas por outros autores (Ozyhar et al., 2012; Mvondo et al., 2017), que concluíram que o material se torna mais dúctil com o aumento do teor de umidade. Tal afirmação corrobora com os resultados obtidos, ou seja, houve uma antecipação da deformação plástica ocorrendo o rompimento da madeira com elevados teores de água presente nas células. Os resultados encontrados por Hering et al. (2012) também estão de acordo com os apresentados na Tabela 1. Esses autores discutem os parâmetros elásticos que, em sua maioria, se mostram menos rígidos com o aumento do conteúdo de umidade no material, com ênfase abaixo do PSF.

Esses resultados ainda podem ser comparados aos encontrados por Tramontina et al. (2013) para Ateleia glazioviana, outra espécie da família Fabaceae, com idade entre 13 e 15 anos, iguais a $84.898 \mathrm{Kgf} \mathrm{cm}^{-2}$ a $12 \%$ de umidade e $56.982 \mathrm{Kgf} \mathrm{cm}^{-2}$ para a madeira úmida. Para Eucalyptus saligna, Haselein et al. (2002) relataram valores de $94.500 \mathrm{Kgf} \mathrm{cm}^{-2}$ para a condição seca a $12 \%$ e $72.900 \mathrm{Kgf} \mathrm{cm}^{-2}$ para a condição úmida, demonstrando superioridade das propriedades de flexão estática dessas madeiras em relação à de ingá.

Cabe ressaltar que o módulo de elasticidade é responsável por caracterizar a rigidez da madeira, a qual deve ser essencialmente considerada em produtos de uso estrutural, já que tem um impacto direto na deformabilidade dos mesmos (Ivkovic et al., 2009). De acordo com Braz et al. (2013), o MOE possui uma importância superior na caracterização tecnológica, tendo em vista a força aplicada perpendicularmente ao eixo longitudinal da peça, podendo dessa forma representar sua resistência.

Com relação ao módulo de ruptura de 1 . marginata, foram evidenciados valores iguais a $660,5 \mathrm{Kgf} \mathrm{cm}^{-2}$ para o material seco a $12 \%$ de umidade e de $481,3 \mathrm{Kgf} \mathrm{cm}^{-2}$ na condição saturada, os quais diferiram dos citados por Tramontina et al. (2013) para A. glazioviana $\left(858 \mathrm{Kgf} \mathrm{cm}^{-2}\right.$ na condição seca e $444 \mathrm{Kgf} \mathrm{^{-2 }}$ para a úmida). Para E.saligna, Haselein et al. (2002) apresentaram valores de $735 \mathrm{Kgf} \mathrm{cm}^{-2}$ e $525 \mathrm{Kgf} \mathrm{cm}^{-2}$ para o material seco e saturado, respectivamente, sendo estes superiores ao da espécie em estudo em relação a essa propriedade mecânica.

Os resultados da razão entre o material saturado e o seco à $12 \%$ de umidade verificados nos testes de flexão estática foram de 1,37 para o MOR e de 1,20 para o MOE, os quais estão próximos aos descritos por Haselein et al. (2002) em estudo com E. saligna, iguais a 1,40 e 1,29 , respectivamente.

As diferenças encontradas para o MOE, quando associado ao teor de umidade, variam de espécie para espécie, pois dependem do comportamento de cada material quando submetido a determinado esforço. Em relação a isso, Freitas (1982) citou que o MOR diminui consideravelmente com o aumento do teor de umidade, enquanto que o MOE resulta em menor alteração, estando de acordo com o obtido no presente trabalho. Ainda, o aumento do teor de umidade reduz as características mecânicas, como a flexão, e a massa específica está diretamente ligada a essas propriedades (Alam et al., 2015).

Com relação à massa específica aparente, os valores citados na Tabela 1 já eram esperados e evidenciaram acréscimo dessa característica com a presença de umidade. Resultados semelhantes foram descritos por Neves Junior et al. (2016) que estudaram madeira de Inga sp. com $15 \%$ de teor de umidade. relatando valor de massa específica aparente igual a $0,758 \mathrm{~g} \mathrm{~cm}^{-3}$, ou seja, entre as médias obtidas. No entanto, a amplitude dos dados observados para a madeira de ingá foi de $0,513 \mathrm{~g} \mathrm{~cm}^{-3}$ até $0,703 \mathrm{~g} \mathrm{~cm}^{-3}$, sendo semelhante à determinada por Eleotério et al. (2015) que encontraram valores entre $0,421 \mathrm{~g} \mathrm{~cm}^{-3} \mathrm{e} 0,638 \mathrm{~g} \mathrm{~cm}^{-3}$ de massa específica aparente a $12 \%$ de umidade em diferentes espécies de eucalipto.

De acordo com a classificação de Carvalho (1996), levando-se em consideração a média obtida de massa específica aparente a $12 \%$ igual a $0,576 \mathrm{~g} \mathrm{~cm}^{-3}$, a madeira de I. marginata pode ser descrita como moderadamente pesada.

A análise de correlação entre as condições de umidade em que o material foi submetido e as propriedades analisadas pode ser observada na Tabela 2 . De maneira 
geral, observou-se grau de dependência estatística linear entre as variáveis, demonstrando a relação existente entre a propriedade física e as características mecânicas.

Tabela 2. Coeficiente de correlação de Pearson existente entre as variáveis de flexão estática e massa específica aparente da madeira de Inga marginata.

Table 2. Pearson correlation coefficient between the static bending variables and apparent specific gravity of Inga marginata wood.

\begin{tabular}{cccc}
\hline Variável & MOE & TLP & MEa \\
\hline MOR & $0,78^{* *}$ & $0,93^{* *}$ & $0,41^{* *}{ }^{-}$ \\
MOE & & $0,84^{* *}$ & $0,37^{*}-$ \\
TLP & & $0,54^{* *}-$ \\
\hline
\end{tabular}

$\mathrm{MOR}=$ módulo de ruptura; $\mathrm{MOE}=$ módulo de elasticidade; TLP $=$ tensão do limite proporcional; $\mathrm{MEa}=$ massa específica aparente; $* * \mathrm{e} *$ significativo ao nível de $1 \%$ e $5 \%$ de probabilidade de erro, respectivamente.

Os resultados da correlação entre as características mecânicas de MOE, MOR e TLP para flexão estática mostraram-se diretamente proporcionais em todas as situações abordadas. No entanto, a análise entre a massa específica aparente e as propriedades mecânicas da madeira demonstraram variação inversamente proporcional.

A variável TLP se destacou na correlação com o MOR, seguido do MOE. Isso pode estar associado à relação existente entre esses parâmetros mecânicos da madeira, ou seja, valores elevados da TLP indicam maior ruptura e elasticidade. A relação inversa entre a resistência mecânica e a massa específica aparente da madeira de I. marginata também está de acordo com o que evidenciaram Lahr et al. (2016). Segundo esses autores, o teor de umidade elevado foi responsável pela redução da propriedade mecânica, assim como, pelo aumento massa específica aparente do material.

\section{Conclusão}

Para as variáveis força máxima, módulo de elasticidade, módulo de ruptura e tensão no limite proporcional, caracterizadas nos ensaios de flexão estática, a madeira de Inga marginata seca à $12 \%$ de umidade demonstra resistência e qualidade superior, quando comparada com o material saturado.

De maneira geral, as amostras tendem a apresentar valores de massa específica aparente menores e maior resistência mecânica, conforme a dessorção até o teor de umidade de equilíbrio de $12 \%$, sendo a madeira, nessa condição, classificada como moderadamente pesada.

Sobretudo, pode-se afirmar que I. marginata ainda é pouco explorado quanto à caracterização de suas propriedades e, com isso, ressalta-se a importância que essa e demais espécies nativas sejam estudadas, possibilitando classificá-las e destiná-las a usos finais adequados.

\section{Referências}

Alam, J. et al. Effect of waterlogged condition on wood properties of Acacia nilotica (L.) debile tree. Bangladesh Journal of Scientific and Industrial Research, v. 50, n. 2, p. 71-76, 2015.

Almeida, D. H. et al. Determinação da rigidez de Pinus elliottii em diferentes teores de umidade por meio de ensaios mecânicos não destrutivos. Scientia Forestalis, v. 44, n. 110, p. 303-309, 2016. DOI: $10.18671 /$ scifor.v44n110.03.

American Society for Testing and Materials. ASTM D 14394: standard test methods for small clear specimens of timber. Philadelphia, 1995.

Braz, R. L. et al. Curva característica de secagem da madeira de Tectona grandis e Acacia mangium ao ar livre. Floresta e Ambiente, v. 1, n. 22, p. 117-123, 2015. DOI: 10.1590/2179-8087.037913.

Braz, R. L. et al. Propriedades físicas e mecânicas da madeira de Toona ciliataem diferentes idades. Floresta, v. 43, n. 4, p. 663-670, 2013. DOI: $10.5380 /$ rf.v43i4.30559.

Bonduelle, G. M. Análise da massa específica e da retratibilidade da madeira de Tectona grandis nos sentidos axial e radial do tronco. Floresta, v. 45, n. 4, p. 671-680, 2015. DOI: 10.5380/rf.v45i4.31991.

Carvalho, A. Madeiras portuguesas: estrutura anatômica, propriedades, utilizações. Lisboa: Instituto Florestal, 1996. v. 1. 340 p. Disponível em: <http://www.estgv.ipv.pt/PaginasPessoais/ jqomarcelo/Tim3/tim3_TP1_Na2.pdf $>$. Acesso em: 28 ago. 2018.

Cezaro, J. A. et al. Propriedades físico-mecânicas da madeira de Chrysophyllum marginatum. Pesquisa Florestal Brasileira, v. 36, n. 86 , p. 135-143, 2016. DOI: 10.4336/2016.pfb.36.86.884.

Eleotério, J. R. et al. Massa específica e retratibilidade da madeira de seis espécies de eucalipto cultivadas no litoral de Santa Catarina. Floresta, v. 45, n. 2, p. 329-336, 2015. DOI: 10.5380/rf.v45i2.34699.

Freitas, A. Potencial de utilização de madeiras em construções. In: ENCONTRO BRASILEIRO EM PRESERVAÇÃO DE MADEIRAS, 1982. São Paulo. Anais... São Paulo: IPT, 1982. p. 1459.

Haselein, C. R. et al. Propriedade de flexão estática da madeira úmida e a $12 \%$ de umidade de um clone de Eucalyptus saligna Smith sob o efeito do espaçamento e da adubação. Ciência Florestal, v. 12, n. 2, p. 147-152, 2002. DOI: 10.5902/198050981689.

Hering, S. et al. Moisture-dependent orthotropic elasticity of beech wood. Wood, Science and Technology, v. 46, n. 5, p. 927-938, 2012. DOI: $10.1007 / \mathrm{s} 00226-011-0449-4$. 
Ivkovic, M. et al. Prediction of wood stiffness, strength, and shrinkage in juvenile wood of radiata pine. Wood Science and Technology, v. 43 , n. 7 , p. $237-257,2009$. DOI: 10.1007/s00226-009-0284-z.

Kollmann, F. F. P. \& Côté, W. A. Principles of wood science and technology. Berlim: Springer-Verlag, 1968. v. 1. 592 p.

Lahr, F. A. R. et al. Avaliação de propriedades físicas e mecânicas de madeiras de Jatobá (Hymenaea stilbocarpa hayne) com diferentes teores de umidade e extraídas de regiões distintas. Revista Árvore, v. 40, n. 1, p. 147-154, 2016. DOI: 10.1590/0100-67622016000100016.

Moriwaki, M. T. et al. Perfil microbiológico e químico de extratos padronizados de Inga marginata. Visão Acadêmica, v. 18, n. 1, p. 4-13, 2017. DOI: 10.5380/acd.v18i1.51279.

Mvondo, R. R. N. et al. Influence of water content on the mechanical and chemical properties of tropical wood species. Results in Physics, v. 7, p. 2096-2103, 2017. DOI: 10.1016/j.rinp.2017.06.025.
Neves Junior, O. F. et al. Propriedades físicas das madeiras de Tectona grandis, Eucalyptus sp., Inga sp. e Artocarpus heterophyllus. In: ENCONTRO BRASILEIRO EM MADEIRAS E EM ESTRUTURAS DE MADEIRAS, 15., 2016, Curitiba. Anais... São Carlos: IBRAMEM, 2016.

Ozyhar, T. et al. Moisture-dependent elastic and strength anisotropy of European beech wood in tension. Journal of Materials Science, v. 47, n. 16, p. 6141-6150, 2012. DOI: 10.1007/s10853-012-6534-8.

SAS Institute. Getting startedwith the SAS learning edition. Cary, 2003. 200 p.

Tramontina, J. et al. Propriedades de flexão estática da madeira úmida e a $12 \%$ de umidade da espécie Ateleia glazioviana Baill. Enciclopédia Biosfera, v. 9, n. 16, p. 1833-1839, 2013.

Trevisan, R. et al. Variação axial e efeito do desbaste na massa específica das árvores centrais de Eucalyptus grandis. Ciência Rural, v. 42, n. 2 , p. $312-318,2012$. 